\title{
Open
}

\section{Enhanced HIV-1 replication in ex vivo ectocervical tissues from post-menopausal women correlates with increased inflammatory responses}

\author{
C Rollenhagen ${ }^{1,2}$ and SN Asin ${ }^{1,2}$
}

Knowledge about early innate immune responses at the mucosal surfaces of the female genital tract is important in understanding the pathogenesis of heterosexual transmission of human immunodeficiency virus type-1 (HIV-1). As estradiol decreases inflammatory responses, we postulated that an estradiol-deficient state such as post-menopause could enhance expression of inflammatory factors that stimulate HIV-1 replication. We compare HIV-1 integration, transcription, and viral p24 release levels among ectocervical tissues obtained from pre- and post-menopausal donors. We detected enhanced HIV-1 p24 release levels in post- compared with pre-menopausal tissues $(P<0.0001)$, but saw no difference in HIV-1 integration. Overall, $100 \%$ of post-menopausal tissues exhibited levels of HIV-1 transcription above background compared with only $60 \%$ of pre-menopausal tissues. Increased HIV-1 transcription was associated with enhanced interleukin (IL)-1 $\beta$, IL-6, monocyte chemotactic protein-1, growth-regulated oncogene- $\alpha$, and interferon- $\gamma$ inducible protein-10 expression. Neutralization and nuclear factor- $\kappa \mathrm{B}$-targeting small-interfering RNA experiments both decreased HIV-1 transcription, suggesting that the early inflammatory response may facilitate HIV-1 replication in ex vivo ectocervical tissues from post-menopausal women.

\section{INTRODUCTION}

Heterosexual transmission of the human immunodeficiency virus type-1 (HIV-1) is the leading cause of virus spread worldwide, with women becoming infected at a higher frequency than men. ${ }^{1}$ The co-factors that enhance a woman's susceptibility to HIV-1 are poorly characterized; however, the reproductive life phase is believed to be relevant in enhancing viral transmission throughout the mucosal surfaces of the female genital tract (FGT). ${ }^{2,3}$ Post-menopausal women have lower estradiol levels, which may increase HIV-1 transmission by modulating early innate immune responses reviewed in the study by Hel et al. ${ }^{4}$ Estradiol, at low levels induces tumor necrosis factor-alpha (TNF)- $\alpha$, interleukin (IL)- $1 \beta$, and IL- 6 expressions, resulting in an inflammatory state that could support HIV-1 replication. ${ }^{5}$ Similarly, greater levels of estradiol during premenopause may decrease immune cell activation and expression of pro-inflammatory cytokines all of which could reduce viral infection. ${ }^{6}$
HIV-1 replication is regulated by a complex network of cytokines and chemokines produced by various hematopoietic and non-hematopoietic cells. These immune modulators influence HIV-1 reverse transcription, viral RNA expression, cellular expression of HIV-1 receptors and co-receptors, as well as migration and activation of HIV-1 target cells, thereby modulating viral infection and replication..$^{7-10}$

Several studies have characterized the kinetics of cytokine expression in the peripheral blood of HIV-infected individuals during the acute phase of infection, and correlated expression levels of these immune factors with plasma HIV-1 RNA. Results from these studies demonstrate enhanced interferon- $\alpha,{ }^{11}$ interferon- $\gamma,{ }^{12} \mathrm{IL}-1 \beta,{ }^{11} \mathrm{IL}-10,{ }^{12}$ interferon- $\gamma$-inducible protein-10 (IP-10), ${ }^{13}$ and regulated upon activation, normal $\mathrm{T}$ cell-expressed and T cell-secreted (RANTES) ${ }^{14}$ expression, whereas TNF- $\alpha$ levels were either enhanced or decreased. ${ }^{11,12}$

In rhesus macaques, a thorough examination of the early mucosal innate immune response after vaginal exposure to the

\footnotetext{
${ }^{1}$ Research Service, V.A. Medical Center, White River Junction, Vermont, USA. ²Department of Microbiology and Immunology, Dartmouth Medical School, Lebanon, New Hampshire, USA. Correspondence: SN Asin (Susana.Asin@Dartmouth.EDU)

These results were presented at the 18th Conference on Retroviruses and Opportunistic Infections, 27 February to 2 March 2011. 
simian immunodeficiency virus (SIV) revealed the predominant induction of pro-inflammatory factors, which correlated with virus replication and temporally preceded the induction of an antiviral response. ${ }^{15}$ Furthermore, pharmacological induction of type I interferons did not blunt SIV vaginal transmission or subsequent viral replication once transmission occurred. ${ }^{16}$

In comparing results between pathogenic and non-pathogenic SIV infection in African green monkeys, an immunosuppressive cytokine profile was associated with lower levels of immune activation and a delayed progression to acquired immunodeficiency syndrome. ${ }^{17}$ Findings in HIV-infected chimpanzees and SIV-infected rhesus macaques suggest that an early and robust inflammatory response may be disadvantageous to the host, due to enhanced T-cell activation and depletion. ${ }^{18,19}$ This conclusion is consistent with results in HIV-1-infected women during the acute phase of the infection, demonstrating an inverse correlation between IL-1 $\beta$, IL-6, and IL-8 expressions in cervicovaginal lavage samples and systemic CD4 ${ }^{+}$T-cell counts. ${ }^{20}$

Although HIV-1-specific adaptive cellular and humoral immune responses have been characterized previously, ${ }^{21-24}$ knowledge about innate immune responses during the acute phase of infection is limited. Cellular responses to SIV appear after the establishment of systemic infection, suggesting that early innate immune responses after sexual exposure are critical to limiting HIV-1 infection and spread at mucosal sites. ${ }^{25}$ Similarly, the lack of association between cervical cellular immune responses to HIV-1 and genital viral shedding implies that early innate immune responses may be relevant to controlling viral replication in the FGT. ${ }^{26}$

As estradiol, at high levels, decreases inflammatory responses, ${ }^{6}$ it is reasonable to postulate that an estradiol-deficient state such as post-menopause will be associated with enhanced secretion of pro-inflammatory factors that stimulate HIV-1 replication. Furthermore, by comparing levels of immune modulators between HIV-infected pre- and post-menopausal tissues, we may identify specific factors that regulate HIV-1 infection and replication at a particular reproductive phase.

We evaluated ex vivo ectocervical tissues obtained from 15 pre- and 16 post-menopausal women for HIV-1 p24 release levels. Furthermore, we assessed HIV-1 integration and levels of viral transcription in ectocervical tissues from 10 additional donors at each reproductive phase. We detected enhanced HIV-1 p24 release levels in supernatants from postcompared with pre-menopausal tissues $(P<0.0001)$. Despite high levels of inter-donor variation, we detected HIV-1 integration in tissues from all donors at each reproductive phase, and saw no differences in the average level of integrated HIV-1 DNA in pre- compared with post-menopausal tissues. Overall, $100 \%$ of post-menopausal tissues exhibited relative levels of HIV-1 transcription above background compared with $60 \%$ of pre-menopausal tissues. In correlating HIV-1 replication with expression of a broad panel of immune modulators with stimulatory effects on HIV-1 transcription, we detected greater constitutive expression of IL- $1 \beta$ and IL- 6 in uninfected tissues obtained from post- compared with pre-menopausal donors. Exposure to HIV-1 strengthened this pro-inflammatory pattern by enhancing IL- $1 \beta$, IL- 6 , monocyte chemotactic protein-1 (MCP-1), growth-regulated oncogene-alpha (GRO- $\alpha$ ), and IP-10 expressions. IL-1 $\beta$-, IL-6-, and MCP-1neutralizing antibodies (Abs), and nuclear factor- $\kappa \mathrm{B}(\mathrm{NF}-\kappa \mathrm{B})$ targeting small-interfering RNA (siRNA) experiments decreased HIV-1 transcription. As these pro-inflammatory factors recruit target cells to mucosal sites of HIV-1 exposure or stimulate viral RNA expression, our findings suggest that enhanced mucosal inflammation during post-menopause may facilitate HIV-1 target cell activation and increase the likelihood of HIV-1 replication and spread at mucosal sites.

\section{RESULTS}

\section{Enhanced HIV-1 p24 release levels in ectocervical tissue explants from post-menopausal women}

Post-menopausal women are believed to be more susceptible to HIV-1 infection, ${ }^{2,3}$ suggesting that low levels of steroid sex hormones may enhance HIV-1 infection or replication in the FGT. To test this hypothesis, we compared HIV-1 p24 release levels ex vivo among ectocervical tissue explants from pre- and post-menopausal women. We focused on the ectocervix, as we have demonstrated that this lower FGT site is more susceptible to HIV-1 infection or more permissive to viral replication compared with the endometrium. ${ }^{27}$

In these experiments, supernatants from HIV-1-infected ectocervical tissue explants from 15 pre- and 16 post-menopausal donors were evaluated for HIV-1 p24 release levels on days 4 and 7 after infection.

By day 4, we detected no differences in HIV-1 p24 release levels in culture supernatants obtained from pre-compared with post-menopausal tissues (Figure 1). Conversely, by day 7, HIV-1 p24 production was higher, a three-fold increase in culture supernatants from post- compared with pre-menopausal tissues $(P<0.0001$; Figure 1). Furthermore, $94 \%$ of post-menopausal tissues demonstrated enhanced HIV-1 p24 release levels compared with $35 \%$ of pre-menopausal tissues from day 4 to day 7 after infection (Figure 1).

The increase in HIV-1 p24 release levels on day 7 after infection suggested the development of a productive viral infection rather than exclusively the release of residual input virus. To address this possibility, ectocervical tissue explants were treated with a single-dose application of the nucleos(t)ide reverse transcriptase inhibitor Tenofovir (TFV) before HIV-1 infection, and evaluated for HIV-1 p24 release levels on days 4 and 7 after infection. By day 4, HIV-1 p24 release levels from TFV-treated tissues were similar to those of untreated controls, demonstrating the exclusive release of residual input virus during the first 4 days after infection (Figure 2). HIV-1 p24 release levels decreased in TFV-treated tissues from day 4 to day 7 after infection, suggesting that a single-dose application of TFV is very effective at preventing HIV-1 infection. In contrast, by day 7 , untreated control tissues had enhanced HIV-1 p24 levels compared with TFV-treated tissues, indicating the release of newly synthesized 


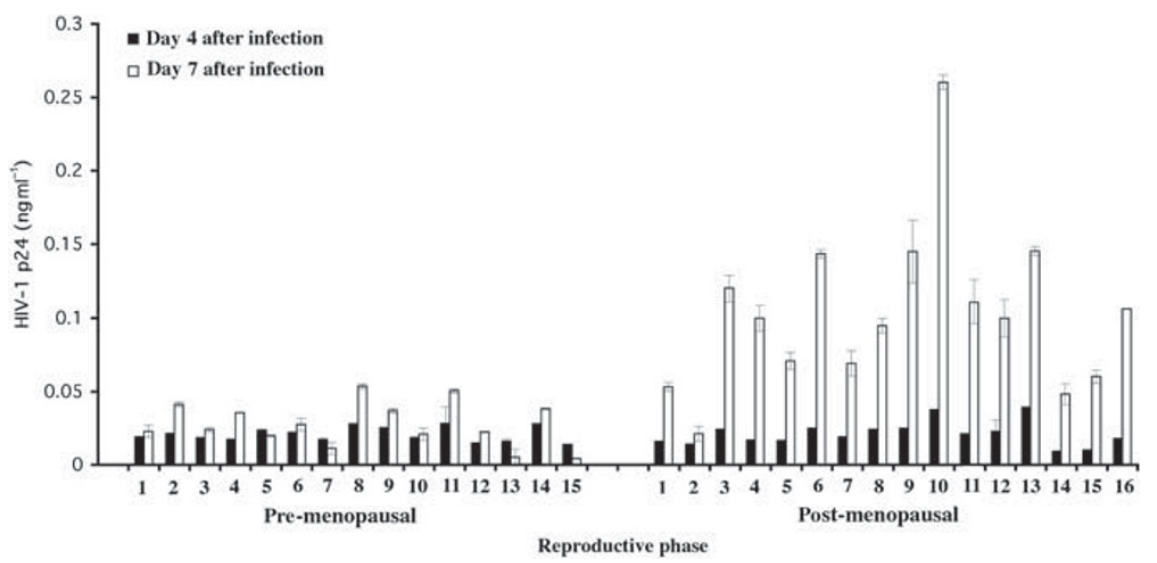

Figure 1 Enhanced HIV-1 p24 release levels in ectocervical tissue explants obtained from post- compared with pre-menopausal women. HIV-1 p24 levels $\left(\mathrm{ng} \mathrm{ml}^{-1}\right)$ in supernatants from HIV-1-infected ectocervical tissue explants on day 4 (black bars) and day 7 (white bars) after infection were measured by HIV-1 p24 ELISA. Viral p24 levels secreted from 15 pre- and 16 post-menopausal individual donors are depicted. Results shown are mean \pm S.d. from three replicates. ELISA, enzyme-linked immunosorbent assay; HIV-1, human immunodeficiency virus type-1.

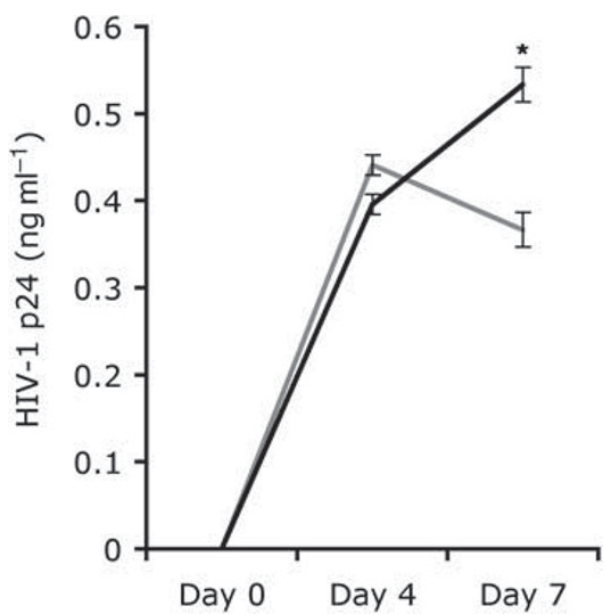

Figure 2 Tenofovir decreases HIV-1 replication in ectocervical tissue explants. HIV-1 p24 release levels $\left(\mathrm{ng} \mathrm{ml}^{-1}\right)$ in supernatants from HIV-1infected ectocervical tissue explants left untreated (black line) or treated with a non-toxic concentration of the NRTI Tenofovir (gray line) on day 4 and day 7 after infection. Results are representative of four donors and are expressed as mean \pm s.e.m. from three replicates. ${ }^{*}$ Indicates a significant difference from the untreated control $(P<0.05)$. HIV-1, human immunodeficiency virus type-1.

virus at this time point, as a function of a productive viral infection (Figure 2).

\section{Greater HIV-1 replication in post-menopausal tissues is unlikely to be due to enhanced HIV-1 integration}

Enhanced HIV-1 p24 release levels could be related to differences in viral entry between pre- and post-menopausal tissues. To test this hypothesis, we evaluated ectocervical tissue explants obtained from 10 additional pre- and post-menopausal donors for integrated HIV-1 DNA by real-time PCR on day 5 after infection. Despite high inter-donor variation, we detected HIV-1 integration in tissues from $100 \%$ of donors at each reproductive phase. Relative levels of integrated HIV-1 DNA in tissues from pre-menopausal donors were similar to those from post-menopausal donors (Figure 3, $P>0.05$ ).

\section{Enhanced HIV-1 transcription in ectocervical tissues from post-menopausal donors}

The presence of similar levels of HIV-1 integration in 100\% of donors at either reproductive phase suggests that enhanced HIV-1 p24 release levels from post-menopausal tissues is likely to be related to differences in the expression of the integrated viral DNA; hence, we evaluated HIV-1 RNA expression in ectocervical tissue explants from the same 10 pre- and 10 post-menopausal donors in whom HIV-1 integration was assessed. We used real-time PCR assays to detect the presence of the HIV-1 early transcripts tat and rev in total cellular RNA isolated on day 5 after infection. Ectocervical tissues from $100 \%$ of post-menopausal donors exhibited relative levels of viral RNA expression above background compared with tissues from $60 \%$ of pre-menopausal donors (Figure 4a). The mean relative level of HIV-1 transcription was $1.2 \pm 0.46$ in post-menopausal donors compared with an average of $0.88 \pm 0.68$ in pre-menopausal donors (Wilcoxon's rank sum $P=0.06$ ). Within each donor, greater levels of HIV-1 integration were not associated with enhanced levels of viral transcription.

To test whether enhanced HIV-1 transcription was related to increased expression of the common leukocyte antigen CD45, we evaluated CD45 transcription in tissues from pre- and postmenopausal donors. We detected high inter-donor variation in CD45 transcription from tissues at either reproductive phase (Figure 4b). The mean relative level of CD45 transcription was $13.67 \pm 3.95$ in post-menopausal tissues compared with an average of $12.86 \pm 3.09$ in pre-menopausal tissues $(P>0.05)$. This result was consistent with our findings from immunofluorescence experiments using ectocervical tissues obtained from two additional pre- and two post-menopausal donors, which confirmed high inter-donor variation in the number of CD45+ intraepithelial and lamina propria leukocytes at either reproductive phase throughout the 7-day culture period (Figure 4c).

As not all CD 45 + cells are HIV-1 targets, we evaluated CD4 transcription in ectocervical tissues from five pre- and seven post-menopausal donors in whom HIV-1 integration and viral transcription was initially assessed. By day 5 , we found similar 


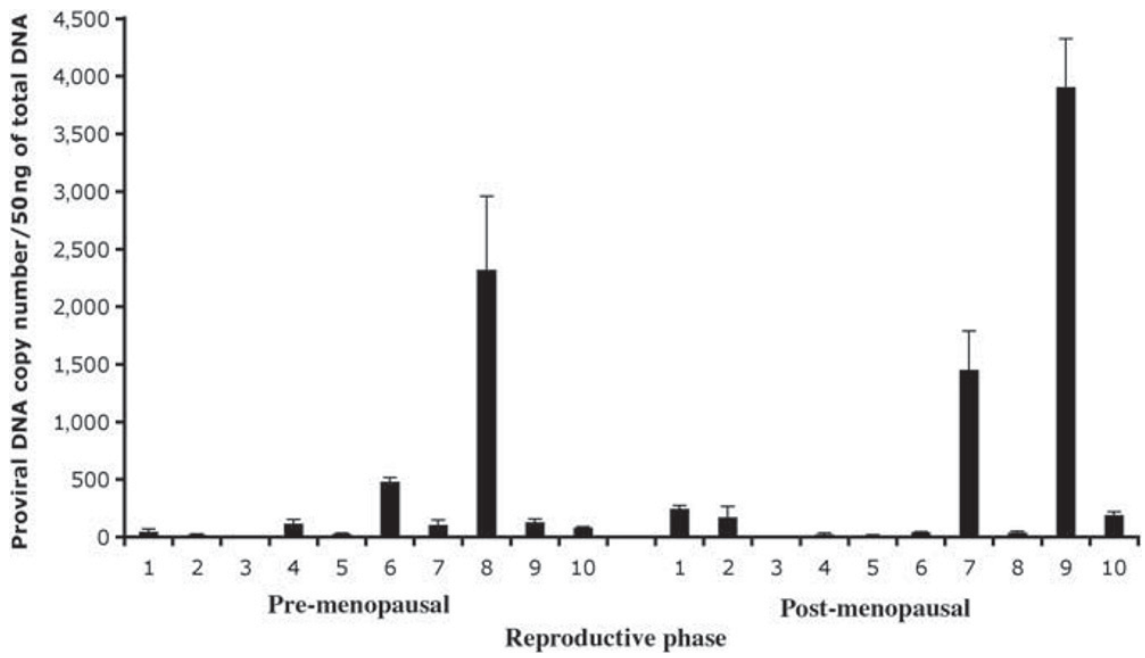

Figure 3 Similar levels of HIV-1 integration in tissues from pre- compared with post-menopausal women. HIV-1 integration was determined by twostep, real-time PCR in ectocervical tissue explants from 10 pre- and 10 post-menopausal donors on day 5 after infection. All data was normalized to $\beta$-actin. Results shown are mean \pm s.d. from three replicates. HIV-1, human immunodeficiency virus type-1.

CD4 expression levels in both pre- and post-menopausal donors (data not shown).

\section{Greater levels of pro-inflammatory cytokines in ectocervical tissues from post-menopausal women}

Our results thus far suggest that differences in viral RNA expression are not related to variations in the number of HIV-1 target cells, but are rather the result of an indirect mechanism due to the secretion of soluble factors that stimulate viral transcription. To determine whether changes occur in the constitutive or HIV-1-induced expression of immune factors that stimulate HIV-1 transcription, we evaluated culture supernatants from ectocervical tissues of pre- and post-menopausal donors for patterns of pro-inflammatory cytokine expression. We detected greater constitutive IL- $1 \beta$ and IL- 6 expression in uninfected ectocervical tissues from post- compared with pre-menopausal women (Figure 5a). In comparing results between reproductive phases, we found enhanced IL- $1 \beta$ and IL- 6 expression in HIV-infected ectocervical tissues from post- compared with pre-menopausal women. In contrast, HIV-1 infection significantly upregulated TNF- $\alpha$ levels in tissues from either reproductive phase (Figure 5b).

\section{Enhanced levels of pro-inflammatory chemokines in ectocervical tissues from post-menopausal women}

HIV-1 infection induced a distinct pattern of pro-inflammatory cytokine expression in post-menopausal tissues; hence, we next addressed whether pro-inflammatory chemokines display a similar pattern of regulation. We compared levels of the CCchemokine MCP-1, and the CXC-chemokines GRO- $\alpha$, IP-10, and IL-8 among ectocervical tissues obtained from pre- and post-menopausal women. Constitutive expression of MCP-1, GRO- $\alpha$, and IP- 10 was similar among supernatants from uninfected pre- and post-menopausal donors (Figure 6a). HIV-1 infection significantly enhanced GRO- $\alpha$ and IP-10 expression, as demonstrated by greater levels of these pro-inflammatory chemokines in supernatants from ectocervical tissues at either reproductive phase after their exposure to HIV-1 (Figure 6a).

In comparing results between reproductive phases, we found enhanced secretion of MCP-1 and GRO- $\alpha$ from ectocervical tissues of post- compared with pre-menopausal women throughout the 7-day culture period (Figure 6a). IP-10 levels were greater in post- than in pre-menopausal tissues only on day 1 after infection (Figure 6a). Constitutive IL-8 levels were similar between supernatants from uninfected pre- and postmenopausal donors, and remained the same after tissue exposure to HIV-1 (Figure 6b).

\section{IL-1 $\beta-$, IL-6-, and MCP-1-neutralizing Abs decrease HIV-1 transcription in ectocervical tissue explants}

To further define the role of the tissue inflammatory response in regulating HIV-1 replication, we conducted blocking experiments in which ectocervical tissue explants were treated with anti-IL-6-, anti-IL-1 $\beta$-, or anti-MCP-1-neutralizing Abs or the isotype control before exposure to HIV-1, and evaluated for HIV-1 transcription on day 5 after infection. We detected a significant decrease (87\% inhibition) in HIV-1 transcription in anti-IL-6-treated tissues compared with isotype control in $100 \%$ of donors (Figure 7a), whereas neutralization of IL-1 $\beta$ and MCP-1 was effective in decreasing HIV-1 transcription only in $40 \%$ of donors (Figure $\mathbf{7 b}$ and $\mathbf{c}$ ). Taken together, our results suggest that IL- $1 \beta$, IL- 6 , and MCP-1 modulate HIV- 1 transcription in ectocervical tissue explants.

\section{NF- $\mathrm{kB}$-targeting siRNA decreases HIV-1 transcription in ectocervical tissue explants}

Our results suggest that enhanced IL- $1 \beta$, IL- 6, MCP- 1 , GRO- $\alpha$, and IP-10 expressions contribute to the greater levels of HIV-1 replication in ectocervical tissues from post-menopausal women. As blocking IL-1 $\beta$ and MCP-1 expressions decreased 

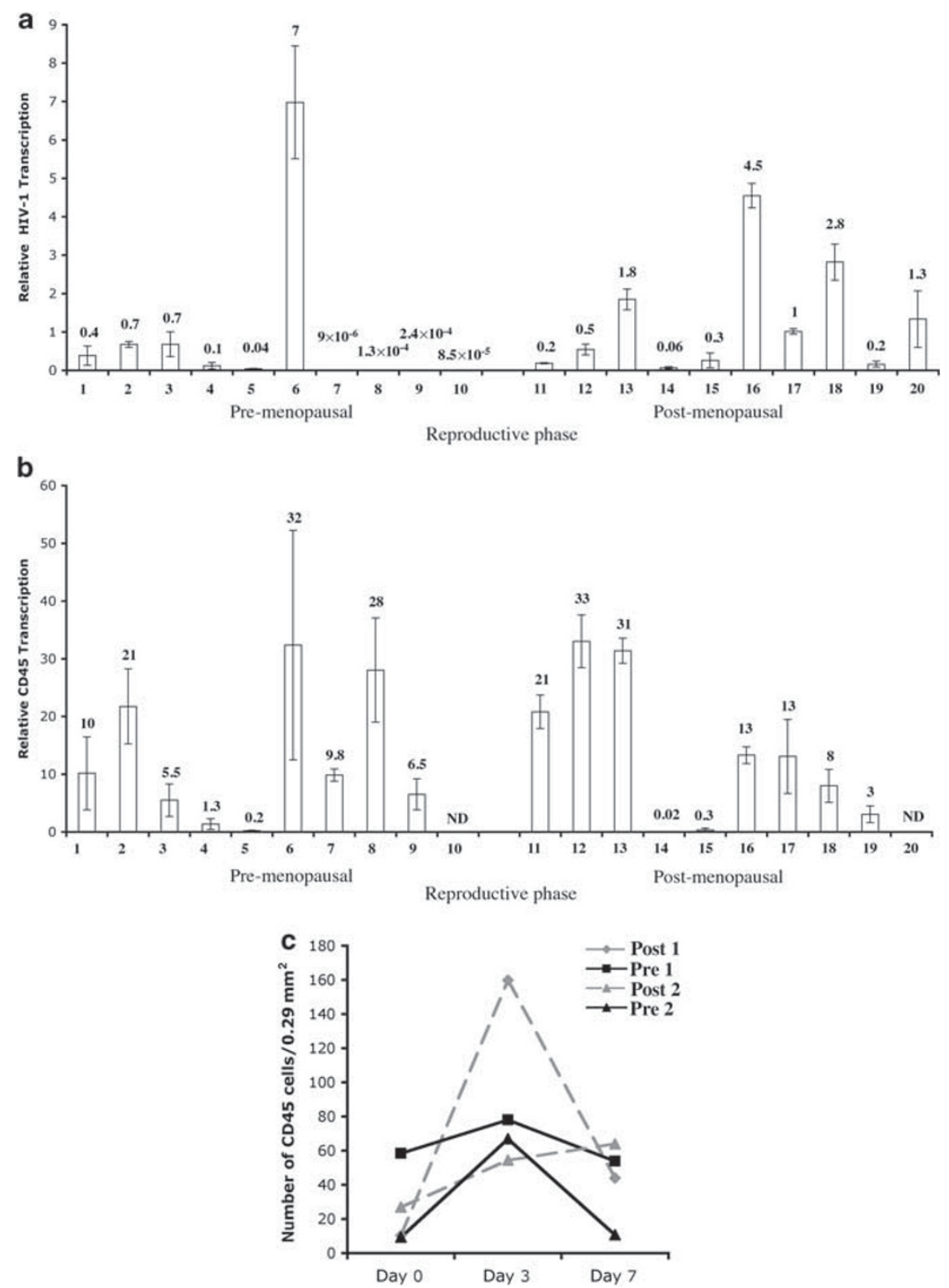

Figure 4 Enhanced HIV-1 transcription in post-menopausal women. (a) Levels of HIV-1 transcription and (b) transcription levels of the leukocyte common antigen CD45 in HIV-1-infected ectocervical tissue explants were quantified by real-time PCR on day 5 after infection. The primers used for HIV-1 transcription target sequences of the HIV-1 early transcripts tat and rev to detect newly synthesized multiply spliced HIV-1 RNA. Transcription values from 10 pre- and 10 post-menopausal donors are depicted. All data were normalized to GAPDH. Results shown are mean \pm s.d. from three replicates. (c) Number of CD45 + cells in pre- (solid black line) and post-menopausal donors (dashed gray line). Intraepithelial and lamina propria CD $45+$ cells per $0.29 \mathrm{~mm}^{2}$ of tissue area were evaluated by immunofluorescence immediately after tissue isolation (day 0 ) and on days 3 and 7 after infection. Results shown are mean \pm s.d. from two donors at each reproductive phase. GAPDH, glyceraldehyde-3-phosphate dehydrogenase; HIV-1, human immunodeficiency virus type-1.

HIV-1 transcription in $40 \%$ of donors, we used siRNA assays to determine the impact of downregulating NF- $\kappa \mathrm{B}$ expression on HIV-1 transcription. Most of the inflammatory factors are induced by NF- $\mathrm{\kappa B}$ or stimulate HIV-1 gene expression by activating this cellular transcription factor. ${ }^{28}$

In these experiments, ectocervical tissue explants from six additional donors were treated with a non-targeting or a NF- $\kappa \mathrm{B}$-targeting siRNA for $48 \mathrm{~h}$ and then infected with HIV-1 for an additional $24 \mathrm{~h}$. Relative levels of HIV-1, NF- $\kappa B$, and CD4 transcription were evaluated on days 2 and 4 after infection. On day 2, NF- $\kappa \mathrm{B}$-targeting siRNA decreased HIV-1 and NF- $\kappa \mathrm{B}$ transcription in all donors compared with tissues treated with non-targeting siRNA (Figure 8a, $P<0.05$ ). Although the decrease in NF- $\kappa \mathrm{B}$ transcription was not sustained through day 4, results from HIV-1 transcription were variable, as three donors demonstrated lower levels of viral RNA expression in tissues treated with NF- $\kappa \mathrm{B}$-targeting compared with non-targeting siRNA (Figure $\mathbf{8 b}, P<0.05$ ); whereas the other 

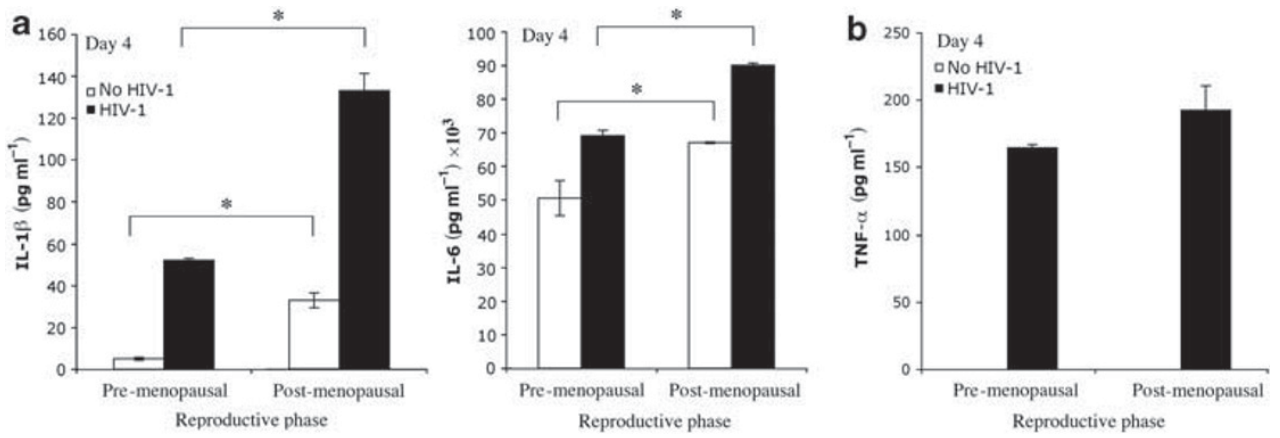

Figure 5 Enhanced secretion of IL-1 $\beta$ and IL-6 in HIV-1-infected and uninfected ectocervical tissues from post-menopausal women. Supernatants from uninfected (white bars) and HIV-1-infected (black bars) ectocervical tissue explants from pre- and post-menopausal women were collected on days 1,4 , and 7 after infection and evaluated for IL-1 $\beta$, IL-6, and TNF- $\alpha$ levels by multiplex Luminex assays. The day after infection with the highest level of secretion is shown. Results shown are mean \pm s.d. from 3 replicates from supernatants pooled from 15 pre- and 16 post-menopausal donors. ${ }^{*}$ Represents statistically significant differences between pre- and post-menopausal women $(P<0.05)$. HIV-1, human immunodeficiency virus type- 1 ; IL, interleukin; TNF- $\alpha$, tumor necrosis factor-alpha.

three demonstrated no differences in viral RNA expression in tissues treated with NF- $\kappa B$-targeting and non-targeting siRNA (Figure 8b).

The decrease in HIV-1 transcription was not related to a decrease in expression levels of the HIV-1 receptor CD4, as we detected similar CD4 transcription in tissues treated with NF- $\kappa B$-targeting or non-targeting siRNA through day 4 after infection (Figure 8a and $\mathbf{b}$ ).

These findings demonstrate a transient NF- $\kappa \mathrm{B}$-mediated decrease in HIV-1 transcription in ectocervical tissue explants within the first 4 days of infection.

\section{DISCUSSION}

New HIV-1 infections are increasing in US women over the age 50 years, mainly through heterosexual transmission, but little is known about specific factors that regulate HIV-1 replication in women this age. ${ }^{29}$ We demonstrated enhanced HIV-1 p24 release levels from ex vivo ectocervical tissue explants from post- compared with pre-menopausal women. By evaluating early post-entry and late transcriptional events in the virus life cycle, we found similar levels of HIV-1 integration in tissues obtained from women at either reproductive phase, but detected greater levels of viral transcription in post- compared with pre-menopausal tissues. These results indicate enhanced viral replication at this reproductive phase, and are consistent with findings from human epidemiological studies demonstrating higher HIV-1 RNA levels in the peripheral blood of postmenopausal women. ${ }^{30}$

It is interesting that we found no correlation between HIV-1 integration and viral RNA expression, suggesting that regulation of transcriptional rather than entry/post-entry events in the virus life cycle is more relevant to defining the kinetics of viral release.

Enhanced HIV-1 transcription was associated with increased IL-1 $\beta$, IL-6, MCP-1, GRO- $\alpha$, and IP-10 expressions in postmenopausal tissues. Although correlative, our findings suggest that this pattern of pro-inflammatory cytokines and chemokines expression may enhance HIV-1 replication ex vivo. IL-1 $\beta$, IL-6,
MCP-1, and IP-10 levels are increased in the peripheral blood and central nervous system of HIV-1-infected individuals, and have proven effective at inducing HIV-1 gene expression reviewed in the study by Herbein et al. ${ }^{31}$ Thus, our findings suggest that post-menopause is associated with enhanced secretion of pro-inflammatory factors that stimulate HIV-1 replication in the FGT.

Constitutive expression of these pro-inflammatory factors was detected in supernatants from uninfected tissue explants during the first $24 \mathrm{~h}$ of tissue isolation. TNF- $\alpha$ and IP- 10 expression was induced, whereas IL-1 $\beta$, IL- $6, \mathrm{MCP}-1$, and GRO- $\alpha$ secretion was enhanced after tissue exposure to HIV-1. As FGT epithelial cells secrete innate immune factors in vitro, and are most likely the first cell population to interact with the virus, ${ }^{32}$ our findings suggest an immune response consistent with the sequential activation of epithelial cells and submucosal leukocytes.

IL- $1 \beta$ and IL- 6 levels in cervicovaginal lavage samples obtained from HIV-infected women have been linked to increased viral genital shedding, implying that these pro-inflammatory factors induce HIV-1 gene expression in the FGT. ${ }^{33-35}$ Thus, we sought to determine whether IL- $1 \beta$ and IL- 6 expression was enhanced in post-menopausal tissues. Both constitutive and HIV-induced levels of IL- $1 \beta$ and IL- 6 were significantly increased in postcompared with pre-menopausal tissues. Furthermore, results from our neutralization experiments demonstrated an $87 \%$ reduction in HIV-1 transcription in $100 \%$ of ectocervical tissues treated with an anti-IL- 6 blocking $\mathrm{Ab}$, whereas neutralizing IL- $1 \beta$ led to a $50 \%$ reduction in viral transcription in $40 \%$ of the tissues compared with isotype control. As these immune modulators are induced by NF- $\mathrm{KB}$, and stimulate HIV-1 gene expression primarily by activating this cellular transcription factor, our findings suggest that the early tissue inflammatory response increases HIV-1 transcription, thereby enhancing viral replication. This hypothesis is consistent with our findings demonstrating that addition of recombinant ( $r$ ) human IL-6 at concentrations normally found within the ectocervix enhances HIV-1 RNA expression. ${ }^{27}$ Thus, under settings of a complex network of inflammatory and counter-regulatory tissue 

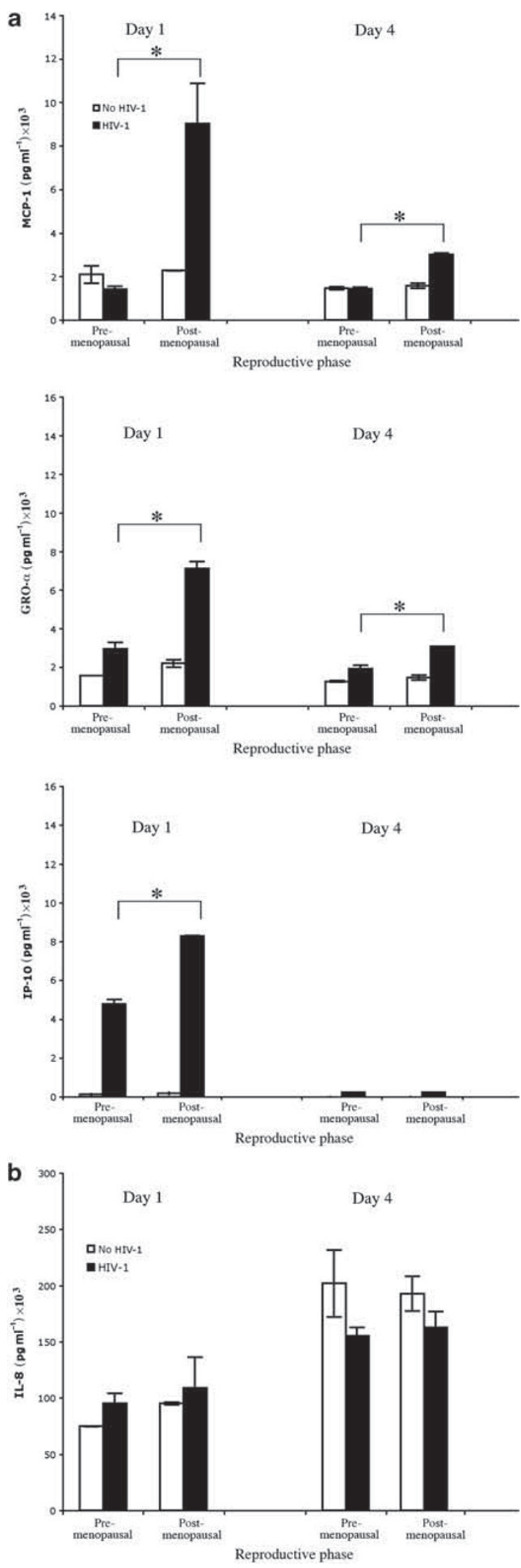

responses, our findings suggest a positive association between IL- $1 \beta$ and IL- 6 expression and HIV-1 replication at primary sites of mucosal transmission.

In addition to pro-inflammatory cytokines, HIV-1 replication is regulated by a complex network of chemotactic factors. By comparing the expression of chemokines with stimulatory effects on viral replication, we detected enhanced MCP-1, GRO- $\alpha$, and IP-10 expressions in supernatants from HIV-infected post- compared with pre-menopausal tissues. Furthermore, blocking MCP-1 expression resulted in a 50\% reduction in HIV-1 transcription levels in $40 \%$ of donors. These chemokines recruit monocytes, neutrophils, dendritic cells, and memory T cells to sites of infection, and have been associated with increased plasma viral load, suggesting their potential to enhance HIV-1 replication in the peripheral blood. ${ }^{36,37}$ As we cannot evaluate recruitment of HIV-1 target cells in tissue explants, the potential of MCP-1, GRO- $\alpha$, and IP- 10 in enhancing HIV-1 replication in post-menopausal tissues may result from activation of immune cells. Thus, our findings underscore the role of chemokines in sustaining HIV-1 replication in the lower FGT.

It is interesting that constitutive IL-8 expression was similar in pre-compared with post-menopausal tissues, and was not enhanced after tissue exposure to HIV-1. Exogenous addition of rIL-8 decreases HIV-1 transcription and viral p24 release in ectocervical tissue explants. ${ }^{38}$ Thus, any potential IL-8-inhibitory effect on HIV-1 replication may have been prevented by the lack of its induction by HIV-1.

Immune activation drives HIV-1 replication. ${ }^{39}$ This conclusion is supported by findings in rhesus macaques wherein the early induction of a pro-inflammatory response was temporally associated with increased levels of viral replication. ${ }^{15}$ Thus, the burst of pro-inflammatory cytokines and chemokines production early after infection could have enhanced viral replication in post-menopausal tissues. Furthermore, results from our siRNA experiments demonstrated that downregulating $N F-\kappa B$, one of the main modulators of the inflammatory response decreased HIV-1 transcription in ectocervical tissues, suggesting that decreasing the tissue inflammatory response is a feasible approach to reduce HIV-1 replication at mucosal sites of viral exposure.

Immune activation by HIV-1 eventually leads to CD $4+$ T-cell depletion; ${ }^{40,41}$ hence, changes in this cell population may

Figure 6 Enhanced levels of MCP-1, GRO- $\alpha$, and IP-10 in HIV-1-infected ectocervical tissues from post-menopausal women. Supernatants from uninfected (white bars) and HIV-1-infected (black bars) ectocervical tissue explants from pre- and post-menopausal women were collected on days 1,4 , and 7 after infection and evaluated for (a) MCP-1, GRO- $\alpha$, IP-10 and (b) IL-8 levels by multiplex Luminex assays. Levels of secretion during a 4-day period are shown. Results shown are mean \pm s.d. from 3 replicates from supernatants pooled from 15 pre- and 16 post-menopausal donors. *Represents statistically significant differences between pre- and post-menopausal women $(P<0.05)$. GRO- $\alpha$, growth-regulated oncogene- $\alpha$; HIV-1, human immunodeficiency virus type- 1 ; IP-10, interferon- $\gamma$-inducible protein-10; MCP-1, monocyte chemotactic protein-1. 

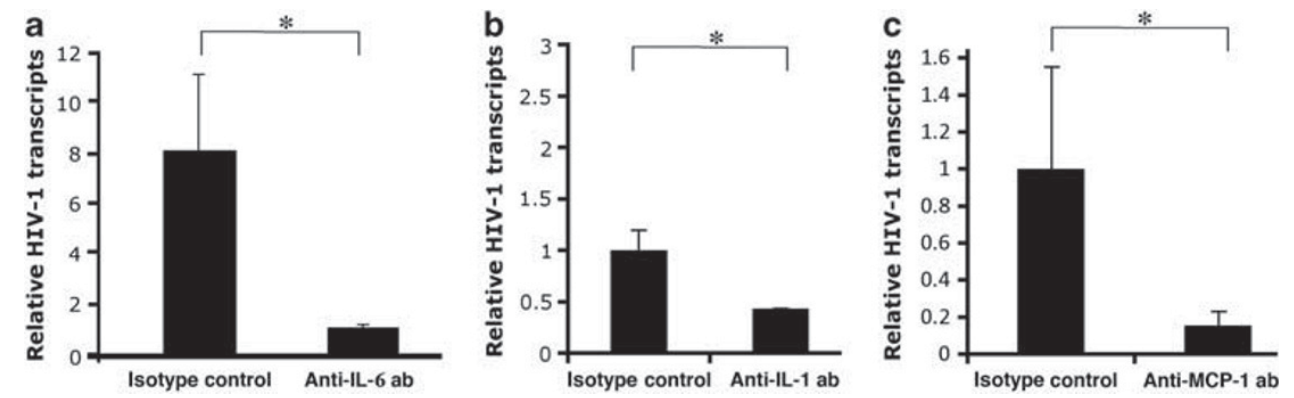

Figure 7 Neutralization of pro-inflammatory cytokines and chemokines decreases HIV-1 transcription in ectocervical tissue explants. Levels of HIV-1 transcription in ectocervical tissue explants treated with an (a) anti-IL-6-, an (b) anti-IL-1 $\beta-$, or an (c) anti-MCP-1-neutralizing antibody or the isotype control were quantified by real-time PCR on day 5 after infection. Results were normalized to GAPDH and are shown as means \pm s.d. for three replicates. ${ }^{*}$ Represents statistically significant differences between tissues treated with neutralizing Abs and isotypes control $(P<0.05)$. GAPDH, glyceraldehyde-3-phosphate dehydrogenase; HIV-1, human immunodeficiency virus type-1; IL, interleukin; MCP-1, monocyte chemotactic protein-1.
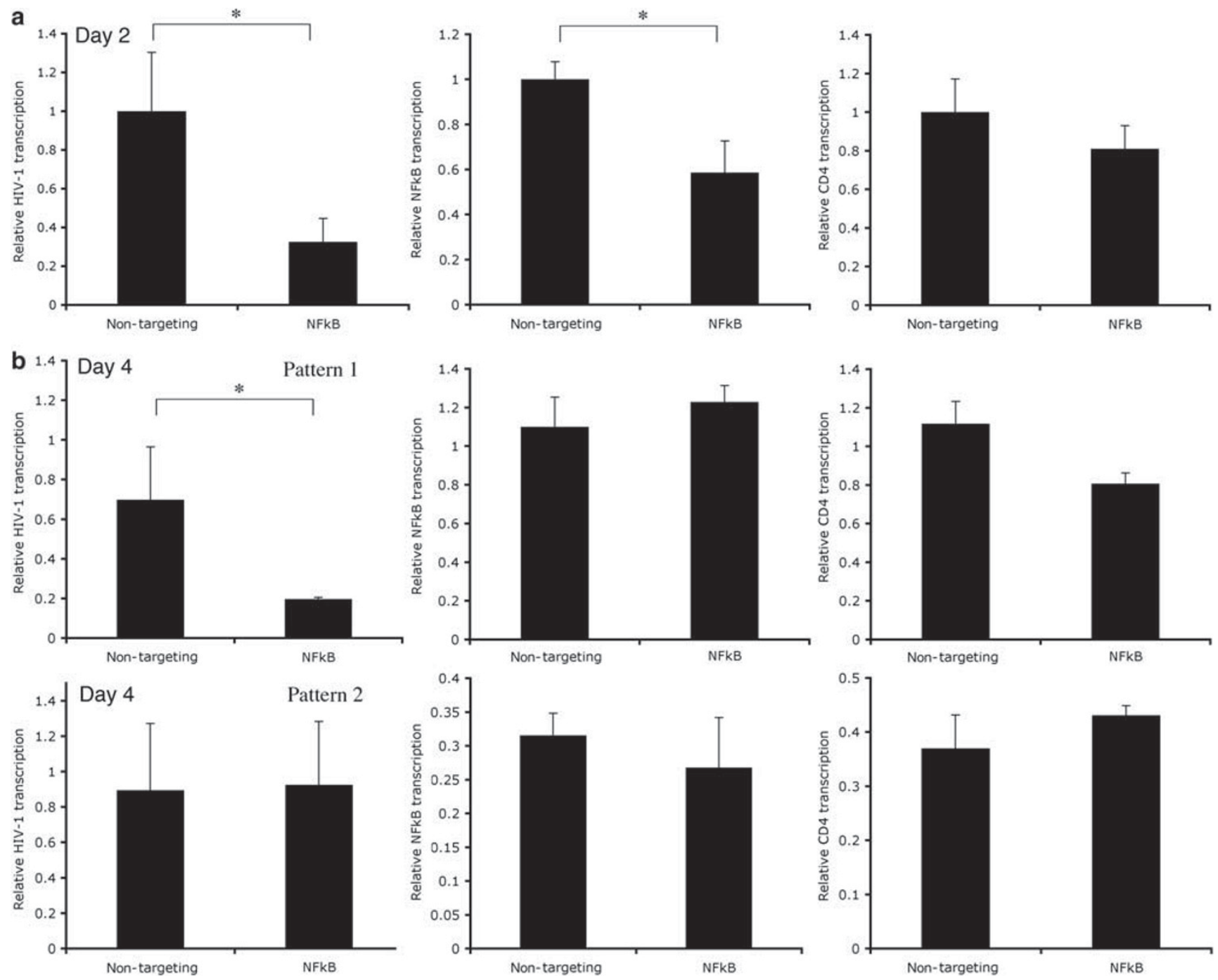

Figure 8 NF-kB-targeting siRNA decreases HIV-1 transcription in ectocervical tissue explants. Relative levels of HIV-1 (left panel), NF- $\mathrm{kB}$ (middle panel), and CD4 (right panel) transcription in HIV-1-infected ectocervical tissue explants treated with non-targeting or NF- $\mathrm{KB}$-targeting siRNAs were quantified by real-time PCR. RNA was extracted on (a) day 2 and (b) day 4 after infection. The primers used for HIV-1 transcription target sequences of the HIV-1 early transcripts tat and rev to detect newly synthesized multiply spliced HIV-1 RNA. Each panel is representative of six experiments. All data were normalized to GAPDH. Results shown are the mean \pm s.d. from three replicates. *Represents statistically significant differences between tissues treated with NF-kB-targeting and non-targeting siRNA $(P<0.05)$. GAPDH, glyceraldehyde-3-phosphate dehydrogenase; HIV-1, human immunodeficiency virus type-1; NF- $\mathrm{kB}$, nuclear factor- $\mathrm{k} B$; siRNA, small-interfering RNA. 
have conditioned the immune response from post-menopausal tissues. However, our results demonstrated similar CD4 expression levels in pre-compared with post-menopausal tissues on day 5 after infection, suggesting that in tissues exposed to HIV-1 $1_{\mathrm{CM} 235}$, T-cell depletion occurs at later time points. Thus, CD4 T-cell depletion is unlikely to have contributed to the changes in cytokine levels described in post-menopausal tissues during a 7-day culture period.

One limitation of the tissue explant model is the migration of several cellular components from the tissues in the first $24 \mathrm{~h}$ of culture, ${ }^{42,43}$ which is expected to alter the innate immune response. Indeed, it is possible that migration of dendritic cells early after infection may have accounted for the lack of interferon- $\alpha$ expression in HIV-1-infected tissues compared with uninfected controls (unpublished data). Thus, the generalization of our conclusions to vaginal transmission of HIV-1 in vivo remains to be defined.

Another confounding factor is the release of residual input virus from the tissues. By treating ectocervical tissues with a single-dose application of TFV before HIV-1 infection, we detected similar levels of HIV-1 p24 in TFV-treated and untreated control tissues on day 4 after infection, indicating the exclusive release of residual input virus at this time point. In contrast, HIV-1 p24 release levels increased from day 4 to day 7 after infection, but only in supernatants from untreated control tissues. This finding demonstrates the release of newly synthesized virus on day 7 after infection, and is consistent with the detection of a specific increase in HIV-1 p24 levels in postcompared with pre-menopausal tissues at this time point. Thus, if our findings were the result of residual virus release, we should have not detected a specific increase in post-menopausal tissues on day 7 , as background levels of residual input virus should be constant across donors as seen on day 4 .

In summary, our results support enhanced replication of an R5-tropic primary HIV-1 isolate in post- compared with premenopausal tissues. This is the first report to postulate that enhanced levels of HIV-1 replication may be accounted for by induction of an early innate inflammatory response. Thus, enhanced mucosal inflammation during post-menopause may lead to increased dissemination of HIV-1 within this primary site of infection.

\section{METHODS}

Tissue samples. Ectocervical tissues were obtained from a total of 59 HIV-seronegative women who were undergoing hysterectomy at the DHMC (Dartmouth-Hitchcock Medical Center) for various benign medical conditions including prolapse and uterine fibroids. Women under 45 years of age were considered pre-menopausal $(n=27)$, and women $\geq 55$ years of age were considered post-menopausal $(n=28) .{ }^{44-46}$ Informed consent was obtained from all donors, and the study protocol was approved by the Committee for the Protection of Human Subjects at Dartmouth College before initiation of sample collection.

Non-polarized ectocervical tissue explants were established in 96-well plates as described previously, ${ }^{27,47}$ and infected with R5-tropic HIV-1 within 3-4h of surgery. Under our culture conditions, tissue explants can be maintained for up to 14 days without a decrease in viability, as determined by an MTS-based microtiter assay (CellTiter 96 Aqueous
One, Promega, Madison WI), and lactate dehydrogenase viability assay (Cytotoxicity Detection Kit, Roche, Indianapolis, IN).

HIV-1 infection. When indicated, tissue explants were exposed to TFV at $100 \mu \mathrm{g} \mathrm{ml}^{-1}$ for $6 \mathrm{~h}$ before viral infection. Tissue explants were infected with $3 \times 10^{6} \mathrm{TCID}_{50}$ of cell-free subtype E R5-tropic HIV-1 ${ }_{\mathrm{CM} 235}$, which is equivalent to $30 \mathrm{ng} \mathrm{ml}^{-1}$ of HIV-1 p24. HIV-1 $\mathrm{CM}_{235}$ has been extensively used in entry and gp120-binding studies, and has been included as a bivalent gp 120 vaccine with $\mathrm{HIV}-1_{\mathrm{SF} 2}$ in phase I/II clinical trials. ${ }^{48,49}$ Furthermore, HIV-1 ${ }_{\mathrm{CM} 235}$ offers the advantage of replicating better in primary cells compared with many other primary R5-tropic viral strains. Viral stocks were generated and titered in cultures of human peripheral

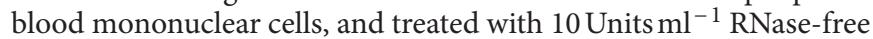
DNase containing $10 \mathrm{mM} \mathrm{MgCl}_{2}$ for $30 \mathrm{~min}$ at room temperature. After overnight incubation at $37^{\circ} \mathrm{C}$, tissue explants were washed to remove residual input virus without disturbing the adherent migratory cells, and cultured for up to 7 days in complete medium. A sample of the culture supernatants was collected after the final wash and evaluated for p24 antigen by enzyme-linked immunosorbent assay (Perkin-Elmer, Boston, MA). Subsequent samples were collected on days 4 and 7 after infection and similarly assayed for HIV-1. At each time point, one-half of the culture supernatant was removed without disturbing the adherent migratory cells, and replenished with an equivalent volume of fresh media. Controls included supernatants from uninfected tissues, washed once, and similarly cultured in complete L-15.

Nucleic acid isolation. Genomic DNA and RNA were isolated from ectocervical tissues on day 5 after infection. Tissue explants, plus any migratory cells, were stored in RNAlater (Ambion, Austin, TX) at $-80^{\circ} \mathrm{C}$ until processing. Tissues were homogenized using a scintered glass homogenizer (Kontes, Vineland, NJ) and the lysate applied to a Shredder column (Qiagen, Valencia, CA). The supernatant was subjected to RNA isolation using the RNeasy-Plus kit (Qiagen). The pellet of the Shredder column was subjected to genomic DNA isolation using the QIAmp DNA mini kit (Qiagen).

HIV-1 integration. HIV-1 proviral DNA was detected by a two-step real-time PCR assay in which the first-round PCR amplifies the DNA sequence between the HIV-1 proviral sequence and the nearest chromosomal ALU elements, and the nested PCR specifically amplifies HIV-1 PCR products pre-amplified in the first round. The first-round amplification was performed for 12 cycles using sense and antisense primers specific for the ALU elements: (5' $5^{\prime}$ TCCC AGCTACTGGGGAGGCTGAGG-3') and (5'-GCCTCCCAAAGTG CTGGGATTACAG-3'), respectively, and the HIV-1 specific primer: (5'-ATGCCACGTAAGCGAAACTCTGGCTAACTAGGGAACCCAC TG-3') extended at its $5^{\prime}$ end with a lambda phage-specific heel sequence. In all, $2 \mu \mathrm{l}$ of this PCR product was used as a template in a secondround amplification, using primers targeting the heel-specific sequence (5'-ATGCCACGTAAGCGAAACT- $3^{\prime}$ ) and the M661 HIV-1 gag sequence ( $5^{\prime}$-CCTGCGTCGAGAGAGCTCCTCTGG-3') of the HIV-1 genome to yield a 159-bp fragment. ${ }^{50} \mathrm{HIV}-1$ integration was calculated by comparison with a standard curve generated by serial dilutions of genomic DNA extracted from $\mathrm{ACH} 2$ cells and expressed as proviral DNA copy number per 50 ng of DNA. Results were normalized to endogenous human $\beta$-actin amplified with the sense primer $\left(5^{\prime}\right.$-CACTCTTCCAGCCTTCCTTCC- $\left.3^{\prime}\right)$ and the antisense primer (5'-CTGTGTTGGCGTACAGGTCT-3').

Gene transcription. Newly synthesized HIV-1 RNA was detected with primers complementary to the flanking sequence of the common splice donor and acceptor sites of the tat and rev genes. In all, $5 \mu \mathrm{g}$ of total RNA was reverse transcribed using Superscript III reverse transcriptase (Invitrogen, Carlsbad, CA) and subjected to real-time PCR using the sense primer ( $5^{\prime}$-TGTAGGCTGACTTCCCGGATGA- $\left.3^{\prime}\right)$ and the 
antisense primer (5'-AGGACTCGGCTTGCTGAGGTG-3') complementary to the genome of $\mathrm{HIV}-1_{\mathrm{CM} 235} \cdot{ }^{27}$ This pair of primers yields a 160-bp product from multiply spliced HIV-1 transcripts. Transcription of the common leukocyte antigen CD45 was evaluated by using the sense primer $\left(5^{\prime}\right.$-CACGGCTGACTTCCAGATATGA-3') and the antisense primer ( $5^{\prime}$-GGTGCTTGCGGGTGAGAAT-3'). This pair of primers yields a 204-bp product from spliced CD45 transcripts. ${ }^{27}$ Transcription of the HIV-1 receptor CD4 was evaluated by using the sense primer (5'-GGCAGTGTCTGCTGAGTGA-3') and the antisense primer (5'-GTGGGCAGAACCTTGATGTTG-3'). This pair of primers yields a 63-bp product. RelA transcription was detected by using the sense primer $\left(5^{\prime}\right.$-CCTGTCCTTTCTCATCCCA- $\left.3^{\prime}\right)$ and the antisense primer (5'-AGCTGCCAGAGTTTCGGTT-3') that yield an 83-bp product. HIV-1, CD45, CD4, and RelA transcription values were normalized to endogenous human GAPDH (glyceraldehyde-3-phosphate dehydrogenase) amplified with the sense primer $\left(5^{\prime}\right.$-GGACCTGACC TGCCGTCTA- $\left.3^{\prime}\right)$ and the antisense primer ( $5^{\prime}$-TGCTGTAGCCA AATTCGTTG-3').

Multiplex Luminex assays. Supernatants from HIV-infected ectocervical tissues were collected on days 1, 4, and 7 after infection and stored at $-80^{\circ} \mathrm{C}$ until processing. To establish a pattern of secretion of immune mediators at each reproductive phase, aliquots of culture supernatants from individual pre- and post-menopausal tissues were pooled, and evaluated for levels of cytokines relevant to the recruitment and activation of innate and adaptive effector cells including TNF $\alpha$, IL-1 $\beta$, IL-6, MCP-1, GRO- $\alpha$, and IP-10 using multiplex Luminex-based-assays (Bio-Rad, Hercules, CA, USA). Supernatants from uninfected tissues from the same donors were used as controls. Unless indicated otherwise, the day after infection with the highest level of secretion is shown.

Bio-Plex Manager software with five-parametric curve fitting (Bio-Rad technical note 2861 at http://www.bio-rad.com) was used for data analysis.

Neutralization experiments. To test whether IL-1 $\beta$, IL-6, and MCP-1 neutralization decreased HIV-1 transcription, ectocervical tissue explants were treated with saturating concentrations $\left(1 \mu \mathrm{g} \mathrm{ml}^{-1}\right)$ of anti-IL-6-, anti-IL-1 $\beta$-, or anti-MCP-1-neutralizing Abs or the isotype control (R\&D Systems, Minneapolis, MN). Abs were added at the time of tissue isolation and maintained in the culture media throughout the experiment. Total tissue RNA was isolated on day 5 after infection and evaluated for HIV-1 transcription as described previously.

NF-KB-silencing experiments. The RelA double-stranded siRNA (5' -UCCAGUGUGUGAAGAAGCGdTdT-3' and 3' -dTdTAGGUCACACACUUCUUCGC-5') was kindly provided by Dr Matthew Vincenti (V.A. Medical Center, White River Junction, VT). The nontargeting siRNA was purchased from Ambion. Interferin-based nanoparticles encapsulating either RelA-targeting or non-targeting siRNA were generated following the manufacturer's instructions (Genesee Scientific, San Diego, CA). In brief, ectocervical tissue explants were exposed to $0.5 \mu$ of interferin pre-incubated with $10 \mu \mathrm{M}$ siRNA in serum-free media for $15 \mathrm{~min}$ at $37^{\circ} \mathrm{C}$. After a 24 -h incubation, the siRNA-interferin complex's concentration was increased onefold and incubated with the tissues for additional $24 \mathrm{~h}$. Ectocervical tissue explants were washed once and then infected with R5-tropic HIV-1. After a 16 -h incubation at $37^{\circ} \mathrm{C}$, tissues were extensively washed to eliminate residual input virus, and fresh media were added back to the cultures. On day 3 , one-half of the culture supernatant was removed without disturbing the adherent migratory cells, and replenished with an equivalent volume of fresh media. Total RNA was isolated on days 2 and 4 after infection and evaluated for HIV-1, $\mathrm{CD} 4$, and NF- $\kappa \mathrm{B}$ transcription as described previously.

Statistical analysis. Analysis of data sets containing two groups was performed by two-sided Student's $t$-test or Wilcoxon's rank-sum test. $P$-values of $<0.05$ were considered significant.

\section{ACKNOWLEDGMENTS}

This work was supported by a Merit Review from the Department of Veterans Affairs (SNA). We thank Dr Ruth Connor, Dartmouth Medical School (DMS), for critical reading of the manuscript. We also thank the Section of Anatomic Pathology, DHMC, Lebanon, NH, for tissue procurement. We thank Dr Jacqueline Smith and Kathleen Smith, Immune Monitoring Laboratory, DMS, for evaluating HIV-uninfected samples by multiplex Luminex assays.

\section{DISCLOSURE}

The authors declared no conflict of interest.

(C) 2011 Society for Mucosal Immunology

\section{REFERENCES}

1. UNAIDS/WHO. Report on Global AIDS Epidemic (UNAIDS, Geneva, 2008).

2. Aaby, P.K. et al. Age of wife as a major determinant of male-to-female transmission of HIV-2 infection: a community study from rural West Africa. AIDS 10, 1585-1590 (1996).

3. European Study Group on Heterosexual transmission of HIV. Comparison of female-to-male and male-to-female transmission of HIV in 563 stable couples. Br. Med. J. 304, 809-813 (1992).

4. Hel, Z., Stringer, E. \& Mestecky, J. Sex steroid hormones, hormonal contraception, and the immunobiology of human immunodeficiency virus1 infection. Endocr. Rev. 31, 79-97 (2010).

5. Straub, R.H. The complex role of estrogens in inflammation. Endocr. Rev. 28, 521-574 (2007).

6. Zang, Y.C., Halder, J.B., Hong, J., Rivera, V.M. \& Zang, J.Z. Regulatory effects of estriol on T cell migration and cytokine profile: inhibition of transcription factor NF-kappa B. J. Neuroimmunol. 124, 106-114 (2002).

7. Kedzierska, K., Crowe, S., Turville, S. \& Cunningham, A.L. The influence of cytokines, chemokines and their receptors on HIV-1 replication in monocytes and macrophages. Rev. Med. Virol. 13, 39-56 (2003).

8. Copeland, K. Modulation of HIV-1 transcription by cytokines and chemokines. Mini Rev. Med. Chem. 5, 1093-1101 (2005).

9. Decrion, A.Z., Dichamp, I., Varin, A. \& Herbein, G. HIV and inflammation. Curr. HIV Res. 3, 243-259 (2005)

10. Kilareski, E.M., Shah, S., Nonnemacher, M.R. \& Wigdahl, B. Regulation of HIV-1 transcription in cells of the monocyte-macrophage lineage. Retrovirology 6, 1-24 (2009).

11. Stacey, A.R. et al. Induction of a striking systemic cytokine cascade prior to peak viremia in acute human immunodeficiency virus type 1 infection in contrast to more modest and delayed responses in acute hepatitis B and C virus infection. J. Virol. 83, 3719-3733 (2009).

12. Norris, P.J., Pappalardo, B., Custer, B., Spotts, G., Hecht, F.M. \& Busch, M.P. Elevations in IL-10, TNF-alpha, and IFN-gamma from the earliest point of HIV type 1 infection. AIDS Res. Hum. Retroviruses 22, 757-762 (2006).

13. Roe, B. et al. Elevated serum levels of Interferon-gamma-inducibleprotein-10 in patients co-infected with hepatitis $\mathrm{C}$ virus and HIV. J. Infect. Dis. 196, 1053-1057 (2007).

14. Reinhart, T.A., Qin, S. \& Sui, Y. Multiple roles for chemokines in the pathogenesis of SIV infection. Curr. HIV Res. 7, 73-82 (2009).

15. Abel, K., Rocke, D.M., Chohan, B., Fritts, L. \& Miller, C.J. Temporal and anatomic relationship between virus replication and cytokine gene expression after vaginal simian immunodeficiency virus infection. J. Virol. 79, 12164-12172 (2005).

16. Wang, Y., Abel, K., Lantz, K., Krieg, A.M., McChesney, M.B. \& Miller, J.C. The Toll-like receptor 7 (TLR7) agonist, imiquimod, and the TLR9 agonist, CpG ODN, induce antiviral cytokines and chemokines but do not prevent vaginal transmission of simian immunodeficiency virus when applied intravaginally to rhesus macaques. J. Virol. 79, 14355-14370 (2005).

17. Kornfeld, C. et al. Anti-inflammatory profiles during primary HIV infection in African green monkeys are associated with protection against AIDS. J. Clin. Invest. 115, 1082-1091 (2005).

18. Silvestri, G. et al. Nonpathogenic SIV infection of sooty mangabeys is characterized by limited bystander immunopathology despite chronic high-level viremia. Immunity 18, 441-452 (2003). 
19. Sopper, S. et al. Impact of simian immunodeficiency virus (SIV) infection on lymphocyte numbers and T cell turnover in different organs of rhesus monkeys. Blood 101, 1213-1219 (2003).

20. Bebell, L.M. et al. Relationship between levels of inflammatory cytokines in the genital tract and CD4+ cell counts in women with acute HIV-1 infection. J. Infect. Dis. 198, 710-714 (2008).

21. Streeck, H. et al. HIV-1-specific CD8+ T cell responses during primary infection are major determinants of the viral set point and loss of CD4+ T cells. J. Virol. 83, 955-959 (2009).

22. Saksena, N.K., Wu, J.Q., Potter, S.J., Wilkinson, J. \& Wang, B. Human immunodeficiency virus interactions with CD8+ T lymphocytes. Curr. HIV Res. 6, 1-9 (2008).

23. Grundström, S. \& Andersson, J. Studies of HIV-associated immune responses in lymphoid compartments. Curr. HIVIAIDS Rep. 3, 32-38 (2006).

24. Frost, S.D., Trkola, A., Günthard, H.F. \& Richman, D.D. Antibody responses in primary HIV-1 infection. Curr. Opin. HIV AIDS 3, 45-51 (2008).

25. Reynolds, M.R. et al. CD8+ T-lymphocyte response to major immunodominant epitopes after vaginal exposure to simian immunodeficiency virus: too late and too little. J. Virol. 79, 9228-9235 (2005).

26. Gumbi, P.P. et al. Impact of mucosal inflammation on cervical human immunodeficiency virus (HIV-1)-specific CD8 T-cell responses in the female genital tract during chronic HIV infection. J. Virol. 82, 8529-8536 (2008).

27. Asin, S.N., Eszterhas, S., Rollenhagen, C., Heimberg, A.M. \& Howell, A.L. HIV-1 in women: increased transcription of HIV-1 in ectocervical tissue explants. J. Infect. Dis. 200, 965-972 (2009).

28. Lawrence, T. The nuclear factor NF-kappaB pathway in inflammation. Cold Spring Harb. Perspect. Biol. 1, a001651 (2009).

29. Meditz, A.L. et al. Sex, race, and geographic region influence clinical outcomes following primary HIV-1 infection. J. Infect. Dis. 203, 442-451 (2011).

30. Patterson, K.B., Cohn, S.E., Uyanik, J., Hughes, M., Smurzynski, M. \& Eron, J.J. Treatment responses in antiretroviral treatment-naive premenopausal and postmenopausal HIV-1-infected women: an analysis from AIDS Clinical Trials Group Studies. Clin. Infect. Dis. 49, 473-476 (2009).

31. Herbein, G., Gras, G., Khan, K.A. \& Abbas, W. Macrophage signaling in HIV-1 infection. Retrovirology 7, 1-15 (2010).

32. Fahey, J.V., Schaefer, T.M., Channon, J.Y. \& Wira, C.R. Secretion of cytokines and chemokines by polarized human epithelial cells from the female reproductive tract. J. Immunol. 174, 992-1002 (2005).

33. Mitchell, C. et al. Cervicovaginal shedding of HIV type 1 is related to genital tract inflammation independent of changes in vaginal microbiota. AIDS Res. Hum. Retroviruses 26, 1-5 (2010).

34. Spear, G.T. et al. Positive association between HIV RNA and IL-6 in the Genital Tract of Rwandan Women. AIDS Res. Hum. Retroviruses 24, 973-976 (2008).

35. Sturm-Ramirez, K., Gaye-Diallo, A., Eisen, G., Mboup, S. \& Kanki, P.J. High levels of tumor necrosis factor-alpha and interleukin-1 beta in bacterial vaginosis may increase susceptibility to human immunodeficiency virus. J. Infect. Dis. 182, 467-473 (2000).
36. Ansari, A.W., Bhatnagar, N., Dittrich-Breiholz, O., Kracht, M., Schmidt, R.E. \& Heiken, H. Host chemokine (C-C motif) ligand-2 (CCL2) is differentially regulated in HIV type 1(HIV-1) infected individuals. Int. Immunol. 18, 1443-1451 (2006).

37. Lane, B.R., Strieter, R.M., Coffey, M.J. \& Markovitz, D.M. Human immunodeficiency virus type 1 (HIV-1)-induced GRO-alpha production stimulates HIV-1 replication in macrophages and T lymphocytes. J. Virol. 75, 5812-5822 (2001).

38. Rollenhagen, C. \& Asin, S.N. IL-8 decreases HIV-1 transcription in peripheral blood lymphocytes and ectocervical tissue explants. J. Acquir. Immune Defic. Syndr. 54, 463-469 (2010).

39. Lawn, S.D., Butera, S.T. \& Folks, T.M. Contribution of immune activation to the pathogenesis and transmission of human immunodeficiency virus type 1 infection. Clin. Microbiol. Rev. 14, 753-777 (2001).

40. Lane, H.C. Pathogenesis of HIV infection: total CD4+ T-cell pool, immune activation, and inflammation. Top HIV Med. 18, 2-6 (2010).

41. Mestecky, J., Moldoveanu, Z., Smith, P.D., Hel, Z. \& Alexander, R.C. Mucosal immunology of the genital and gastrointestinal tracts and HIV-1 infection. J. Reprod. Immunol. 83, 196-200 (2009).

42. Fletcher, P. et al. The nonnucleoside revere transcriptase inhibitor UC-781 inhibits human immunodeficiency virus type I infection of human cervical tissue and dissemination by migratory cells. J. Virol. 79, 11179-11186 (2005).

43. Wallace, G.S. et al. Human immunodeficiency virus type 1 nucleocapsid inhibitors impede trans infection in cellular and explant models and protect nonhuman primates from infection. J. Virol. 83, 9175-9182 (2009).

44. Gold, E.B. et al. Factors associated with age at natural menopause in a multiethnic sample of midlife women. Am. J. Epidemiol. 153, 865-874 (2001).

45. Henderson, K.D., Bernstein, L., Henderson, B., Kolonel, L. \& Pike, M.C. Predictors of the timing of natural menopause in the Multiethnic Cohort Study. Am. J. Epidemiol. 167, 1287-1294 (2008).

46. McKinlay, S.M., Brambilla, D.J. \& Posner, J.G. The normal menopause transition. Maturitas 14, 103-115 (1992).

47. Greenhead, P., Hayes, P., Watts, P.S., Laing, K.G., Griffin, G.E. \& Shattock, R.J. Parameters of human immunodeficiency virus infection of human cervical tissue and inhibition by vaginal virucides. J. Virol. 74, 5577-5786 (2000).

48. Doranz, B.J., Baik, S.S. \& Doms, R.W. Use of a gp120 binding assay to dissect the requirements and kinetics of human immunodeficiency virus fusion events. J. Virol. 73, 10346-10358 (1999).

49. Polianova, M.T., Ruscetti, F.W., Pert, C.B. \& Ruff, M.R. Chemokine receptor-5 (CCR5) is a receptor for the HIV entry inhibitor peptide T (DAPTA). Antiviral Res. 67, 83-92 (2005).

50. Brussel, A., Delelis, O. \& Sonigo, P. Alu-LTR real -time nested PCR assay for quantifying integrated HIV-1 DNA. Methods Mol. Biol. 304, 139-154 (2005).

(c) This work is licensed under the Creative Commons SOMERाHIS RESERVED Attribution-NonCommercial-Share Alike 3.0 Unported License. To view a copy of this license, visit http://creativecommons.org/licenses/by-nc-sa/3.0/ 